\title{
Power Density Optimization for Micro Thermoelectric Generators
}

Marc T. Dunham ${ }^{\mathrm{a}}$, Michael T. Barako ${ }^{\mathrm{a}}$, Saniya LeBlanc ${ }^{\mathrm{b}}$, Mehdi Asheghi ${ }^{\mathrm{a}}$, Baoxing Chen ${ }^{\mathrm{c}}$, and Kenneth E. Goodson $^{\mathrm{a} \dagger}$

${ }^{a}$ Department of Mechanical Engineering, Stanford University, Stanford, CA, 94305

${ }^{\mathrm{b}}$ Department of Mechanical Engineering, The George Washington University, Washington, DC, 20052

${ }^{\mathrm{c}}$ Analog Devices, Inc., Norwood, MA, 02062

$\uparrow$ Author to whom correspondence should be addressed: goodson@stanford.edu 


\begin{abstract}
Microfabricated thermoelectric generators ( $\mu$ TEGs) can harvest modest temperature differences to provide reliable solid-state electricity for low-power electronics, sensors in distributed networks, and biomedical devices. While past work on $\mu$ TEGs has focused on fabrication and demonstration, here we derive and explore comprehensive design guidelines for optimizing power output. A new closed-form thermoelectric device model agrees well with the traditional iterative approach. When thermoelectric leg length is limited by thin-film fabrication techniques, a very low $(<10 \%)$ active thermoelectric fill fraction is required to optimize device power output, requiring careful selection of filler material. Parasitic resistance due to electrical interconnects is significant when a small number of thermocouples is used, and this loss can be reduced by increasing the number of thermocouples while decreasing the cross-sectional area of the legs to maintain the same fill fraction. Finally, a discussion of the "incompleteness of $Z T$ " shows that different combinations of thermal conductivity, electrical conductivity, and Seebeck coefficient resulting in the same $Z T$ will result in different device performance and optimization decisions. For $\mu$ TEGs, we show it is best to increase Seebeck coefficient, followed by decreasing thermal conductivity for short leg lengths and increasing electrical conductivity for long leg lengths.
\end{abstract}

\title{
1. Introduction
}

Microfabricated thermoelectric generators ( $\mu$ TEGs) are used to produce electrical power for devices, such as wireless sensors, requiring micro-Watts to milli-Watts of power per device. These generators scavenge thermal energy from waste heat sources that have temperature 
differences or spatial dimensions that are too small for conventional thermodynamic heat engines to effectively utilize. In this domain, only a small fraction of available thermal energy needs to be extracted from the thermal reservoir to achieve a target power output, often without concern for thermal efficiency. In the limit where the heat capacities of heat source and sink are very large, the temperatures of the source and sink are not appreciably perturbed by the small amount of heat drawn through the $\mu$ TEG system, which is dependent on device configuration (i.e. the geometry, interfaces, boundary conditions, etc.). This fixed temperature assumption is an ideality that benefits from the relatively high thermal resistance of a $\mu \mathrm{TEG}$, compared to larger devices for which local temperature perturbations may be a more present concern [1]. Under a fixed temperature assumption, optimization for either maximum thermal efficiency or maximum power output will lead to two different devices $[2,3]$. Since many microfabricated devices, sensors, and actuators have well-defined power and footprint requirements, this work defines and optimizes performance based on electrical power generated by the device rather than thermal efficiency and presents a comprehensive design methodology for microfabricated waste heat scavenging devices.

The field of $\mu$ TEGs emerged in the early/mid-1990s, reducing feature sizes to $\mathrm{mm}$ and $\mu \mathrm{m}$ following decades of successful use of high-temperature, bulk-scale devices for applications such as spacecraft power generation [4]. Microfabricated devices offer an advantage over traditional bulk-processed devices by allowing for streamlined assembly in semiconductor process lines and direct on-chip integration. One of the first discussions of the use of thermoelectric generators for the recovery of "low-grade" waste heat examined mm-scale devices exposed to temperature sources up to $240{ }^{\circ} \mathrm{C}$ [5]. In 1997, Fleurial et al. [6] suggested the use of thin-film fabrication methods for "micropower sources" to accommodate the advance in miniaturized systems, 
particularly for space applications. In the same year, Stordeur and Stark [7] reported on the successful fabrication and testing of a $\mu \mathrm{TEG}$, produced by thin-film sputtering in a planar pattern followed by dicing and vertical assembly. Fleurial et al. [8,9] achieved thicker $(\sim 10-50 \mu \mathrm{m})$ thermoelectric films deposited by electrodeposition for low-power, high-voltage harvesting of small temperature gradients. In 2004, Böttner et al. [10] discussed a novel sandwich-type wafer assembly with interlocking substrates prepared by co-sputtering. Strasser et al. [11] prepared and analyzed a CMOS-compatible generator design based on $\mathrm{Si}$ and $\mathrm{SiGe}$ semiconductors in a design where heat flows laterally in the device. Electrodeposition of bismuth-telluride and nickel-copper devices using polymer molds was reported by Glatz et al. [12] to extend the regime of thin film thermoelectric leg lengths, which is a limiting factor in microfabricated devices.

While significant progress has been made in fabrication and preliminary demonstration of $\mu$ TEGs, there has been little attention on the challenge of optimizing these devices for power output. While optimizing $Z T$ at the materials level has been the focus of much of the thermoelectrics community, these reports may not be complete as the $\mu \mathrm{TEG}$ community strives to improve power generation per unit footprint area for practical applications, a particularly important metric for small thermoelectric modules converting waste heat to electricity. The use of the metric $Z T$ as an indicator of the quality of thermoelectric generators [4,13-15] may not be appropriate for $\mu$ TEGs. This quantity encapsulates the operating temperature and three primary material properties contributing to the thermoelectric rate equations into a dimensionless parameter, the so-called thermoelectric figure-of-merit:

$$
Z T=\frac{S^{2}}{\rho k}\left(\frac{T_{\mathrm{h}}+T_{\mathrm{c}}}{2}\right)
$$


In Eq. (1), $S$ is the Seebeck coefficient (units of $\mu \mathrm{V} \mathrm{K}^{-1}$ ), $\rho$ is the electrical resistivity $(\Omega \mathrm{m}), k$ is the thermal conductivity $\left(\mathrm{W} \mathrm{m}^{-1} \mathrm{~K}^{-1}\right)$, and $T_{\mathrm{h}}$ and $T_{\mathrm{c}}$ are the hot and cold temperatures across the thermoelectric. In reality, an "incompleteness of $Z T$ " exists where a single value of $Z T$ does not uniquely predict power output or provide an absolute roadmap to device optimization due to the many combinations of $S, k$, and $\sigma$ that yield a single $Z T$ value.

The impact of filler material, which surrounds the active thermoelectric material (see Fig. 1) and may exist as a byproduct of manufacturing or intentionally for mechanical stability, is often overlooked despite observed effects on performance [16]. External thermal resistances (i.e. the pathways to heat sources/sinks) play a significant role in the performance of $\mu$ TEGs, and some attention has been given to these effects [17-19]. However, without the inclusion of realistic filler material effects and device design considerations, these analyses are incomplete. Additional factors become important in the realization of a practical generator device, including adjustment of device fill fraction to compensate for fabrication limitations to thermoelectric element length and appropriate selection of number of junctions when accounting for the electrical resistance of interconnects.

The present work develops a comprehensive design methodology for $\mu$ TEG devices, particularly those harvesting energy from small temperature differences, paying close attention to several parameters that are commonly neglected in the existing literature, such as the effects of fill fraction in combination with different filler materials, limitations and workarounds when restricted to short leg lengths, the impact of the number of thermocouples, and the different contributions of the parameters in the figure of merit $Z T$ to device performance. Further, a robust closed-form model for the power output of a $\mu$ TEG is derived, which includes the Peltier effect, 
external thermal resistances, parasitic resistive losses, fill fraction/filler material, and accounts for variable electric loading.

\section{Model}

The analysis presented in this paper considers a thermoelectric generator with heat flowing through the individual thermoelectric elements in parallel and electric current flowing in a serpentine series pattern. Simulations use a thermocouple unit cell that can be repeated to scale up to a full device. The unit cell area and relevant segments of the generator for simulation are illustrated in Fig. 1.

(a)

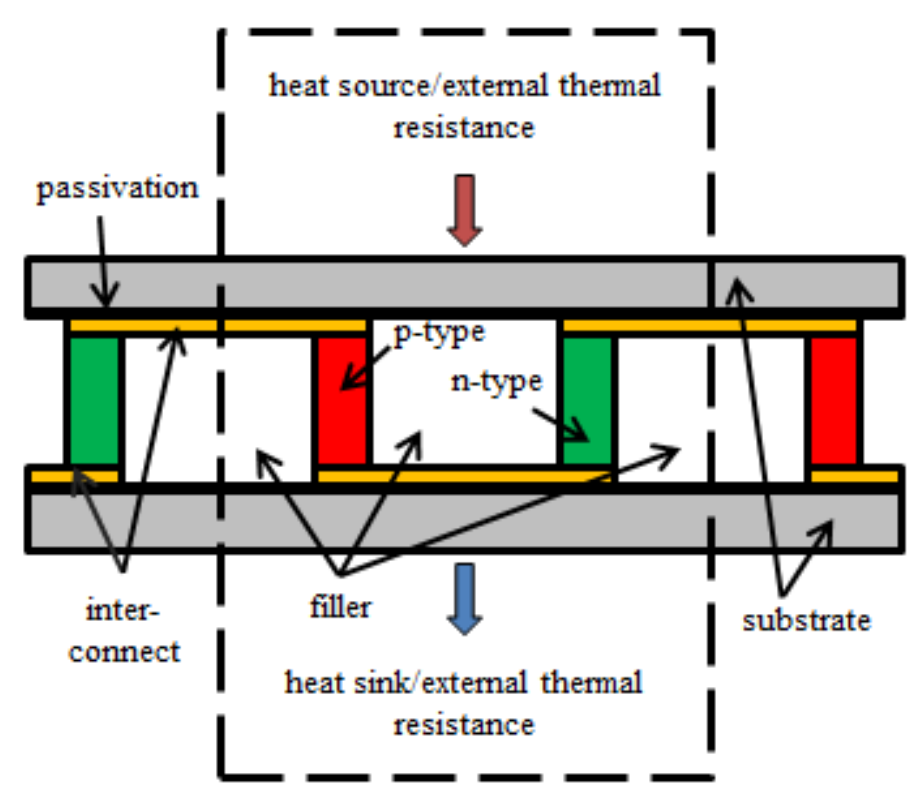

(b) 


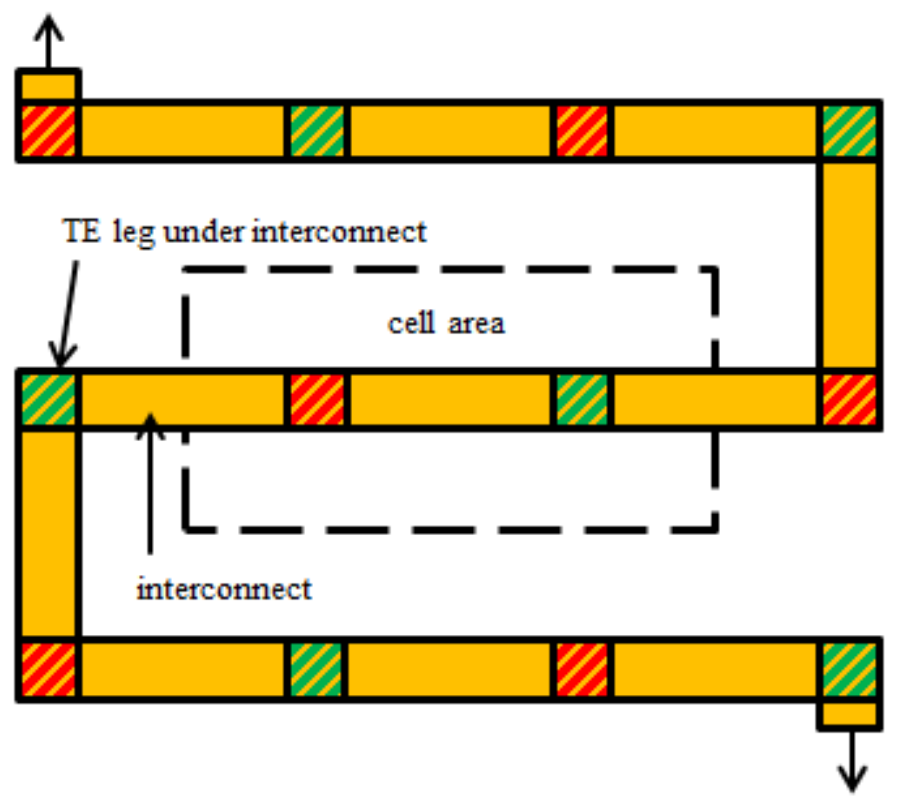

Fig. 1. Diagram of vertically-aligned thermoelectric generator, showing (a) side and (b) top views.

The illustration in Fig. 1 shows both a side view with heat flowing from one substrate to the other and a top-down view with heat flowing into/out of the page. A one-dimensional heat transfer model with parallel heat flows is assumed for the vertical structure shown in Fig. 1, and the thermal and electrical resistance networks are provided in Fig. 2. For simplicity, we assume that the thin passivation layers and high thermal conductivity metal interconnects in series with relatively long and low-conductivity thermoelectric legs do not contribute significantly to the overall thermal resistance. This assumption is confirmed with $3 \mathrm{D}$ finite element analysis.

(a) 

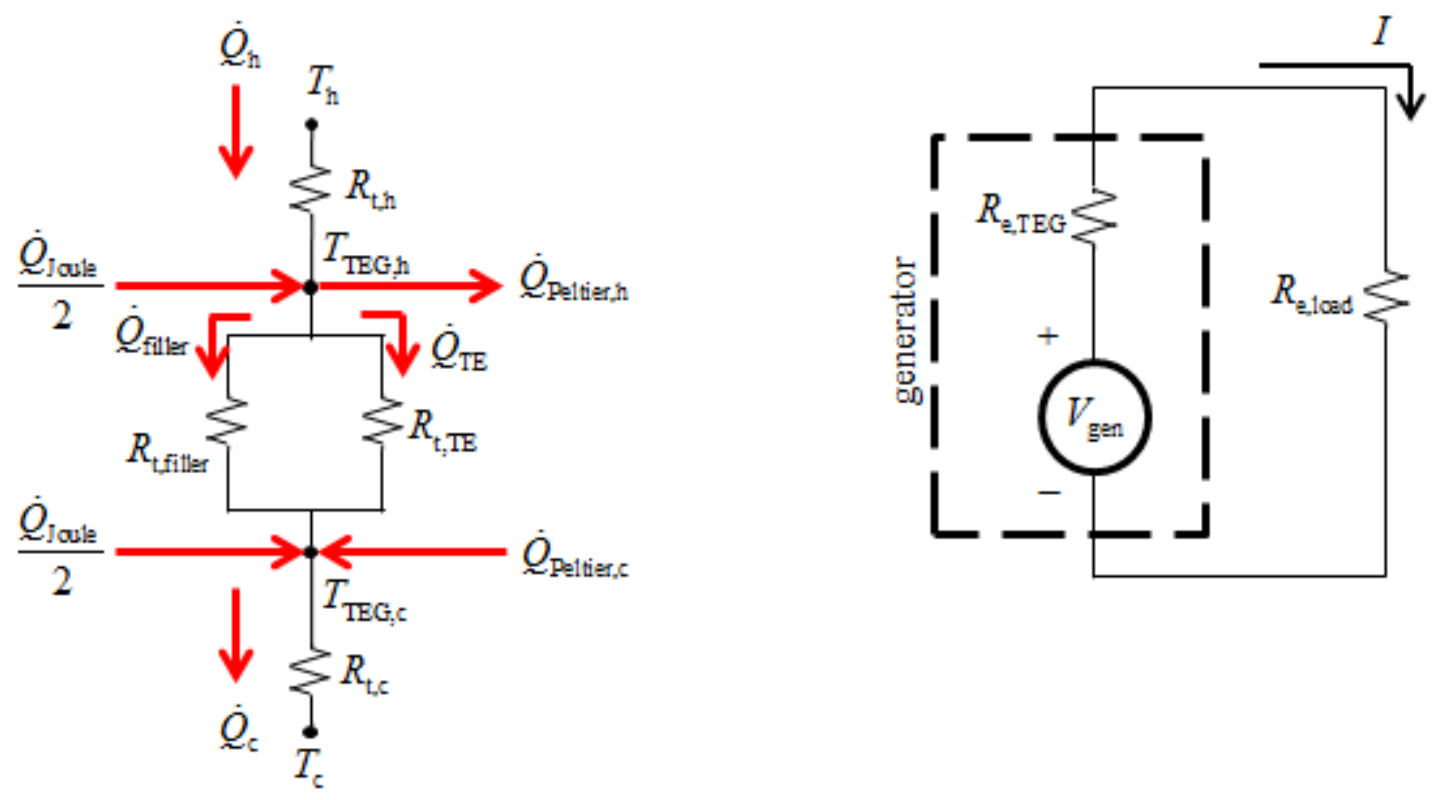

\section{Fig. 2. (a) One-dimensional thermal and (b) electrical resistance networks used to model thermoelectric generators.}

The model accounts for thermal conduction through external thermal resistances $R_{\mathrm{t}, \mathrm{h}}$ and $R_{\mathrm{t}, \mathrm{c}}(e . g$. heat sinks/exchangers), substrate resistances, and parallel conduction through the active thermoelectric material and filler material. This model also includes the effects of Joule and Peltier heating. Joule heating $\left(\dot{Q}_{\text {Joule }}\right)$ is volumetric and well-modeled by introducing half of the total heat at each of the hot and cold junctions of the device [20]. The transfer of heat from the hot junction to the cold junction by the Peltier effect is captured by a removal of heat at the hot end and an addition of heat at the cold end and degrades the performance of thermoelectric devices operating as electric generators. Thomson heat is neglected due to the small temperature gradients encountered in micro-harvesting applications. A nodal energy balance at the hot junction including heat flow from the source $\left(\dot{Q}_{\mathrm{h}}\right)$, heat flow through the thermoelectric material $\left(\dot{Q}_{\mathrm{TE}}\right)$, heat flow through the filler material $\left(\dot{Q}_{\mathrm{filler}}\right)$, heat removed at the hot junction due to the Peltier effect ( $\dot{Q}_{\text {Peltier, }}$ ), and heat added at the cold junction due to Joule heating $\left(\dot{Q}_{\text {Joule }}\right.$ ) is given by 


$$
0=-\dot{Q}_{\mathrm{h}}+\dot{Q}_{\mathrm{TE}}+\dot{Q}_{\mathrm{filler}}+\dot{Q}_{\mathrm{Peltier}, \mathrm{h}}-\frac{\dot{Q}_{\mathrm{Joule}}}{2}
$$

where:

$$
\begin{gathered}
\dot{Q}_{\mathrm{h}}=\frac{T_{\mathrm{h}}-T_{\mathrm{TEG}, \mathrm{h}}}{R_{\mathrm{t}, \mathrm{h}}} \\
\dot{Q}_{\mathrm{TE}}=\frac{T_{\mathrm{TEG}, \mathrm{h}}-T_{\mathrm{TEG}, \mathrm{c}}}{R_{\mathrm{t}, \mathrm{TE}}} \\
\dot{Q}_{\text {filler }}=\frac{T_{\mathrm{TEG}, \mathrm{h}}-T_{\mathrm{TEG}, \mathrm{c}}}{R_{\mathrm{t}, \mathrm{filller}}} \\
\dot{Q}_{\text {Peltier, }}=n_{\mathrm{TC}} S_{\mathrm{net}} T_{\mathrm{TEG}, \mathrm{h}} I \\
\dot{Q}_{\text {Joule }}=I^{2} R_{\mathrm{e}, \mathrm{TEG}}
\end{gathered}
$$

The temperatures of the hot and cold junctions are given by $T_{\mathrm{TEG}, \mathrm{h}}$ and $T_{\mathrm{TEG}, \mathrm{c}}$, respectively. The number of thermocouple junctions is expressed by $n_{\mathrm{TC}}$, and $S_{\text {net }}$ is the net Seebeck coefficient (defined as $S_{\text {net }}=S_{\mathrm{p}}-S_{\mathrm{n}}$ ). The electrical current through and electrical resistance of the generator are $I$ and $R_{\mathrm{e}, \mathrm{TEG}}$, respectively. The parallel thermal resistances of the thermoelectric material and filler material are given by:

$$
\begin{gathered}
R_{\mathrm{t}, \mathrm{TE}}=\frac{l_{\mathrm{TE}}}{k_{\mathrm{TE}} A_{\mathrm{total}} F F} \\
R_{\mathrm{t}, \text { filler }}=\frac{l_{\mathrm{TE}}}{k_{\text {filler }} A_{\text {total }}(1-F F)}
\end{gathered}
$$

where $l_{\mathrm{TE}}$ is the length (or height) of the each thermoelectric leg, $k_{\mathrm{TE}}$ and $k_{\text {filler }}$ are the respective thermal conductivities of the thermoelectric and filler material, $F F$ is the filling fraction of active thermoelectric material, and $A_{\text {total }}$ is the device footprint area.

Similarly, we construct an energy balance at the cold junction:

$$
0=\dot{Q}_{\mathrm{c}}-\dot{Q}_{\mathrm{TE}}-\dot{Q}_{\mathrm{filller}}-\dot{Q}_{\mathrm{Peltier}, \mathrm{c}}-\frac{\dot{Q}_{\mathrm{Joule}}}{2}
$$


where:

$$
\begin{aligned}
\dot{Q}_{\mathrm{c}} & =\frac{T_{\mathrm{TEG}, \mathrm{c}}-T_{\mathrm{c}}}{R_{\mathrm{t}, \mathrm{c}}} \\
\dot{Q}_{\text {Peltier, } \mathrm{c}} & =n_{\mathrm{TC}} S_{\text {net }} T_{\mathrm{TEG}, \mathrm{c}} I
\end{aligned}
$$

The validity of the 1D thermal model (excluding Joule and Peltier contributions) is checked with a 3D finite element simulation in COMSOL Multiphysics. The 3D simulation includes two 100 $\mathrm{nm} \mathrm{Si}_{3} \mathrm{~N}_{4}$ passivation layers (implemented first as narrow domain elements and then using the Thin Layer resistance feature with negligible discrepancy) and $1 \mu \mathrm{m}$ tall Au interconnects matched flush to the sides of the thermoelectric legs. The average temperature of the two thermoelectric (TE) legs is calculated at the hot and cold junctions, and the temperature difference is compared to that calculated using the 1D heat transfer approximation. The results are compared in Fig. 3 for $F F=0.01$ and 0.25 , and 4 different filler materials (vacuum, air, polyimide, and $\mathrm{SiO}_{2}$ ). 


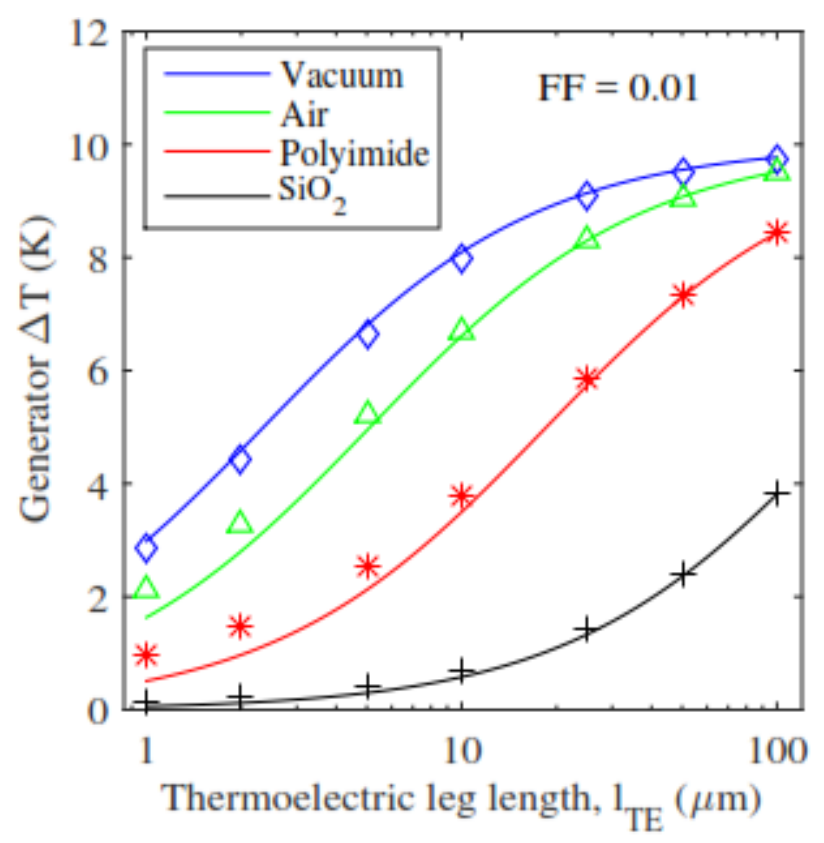

(b)

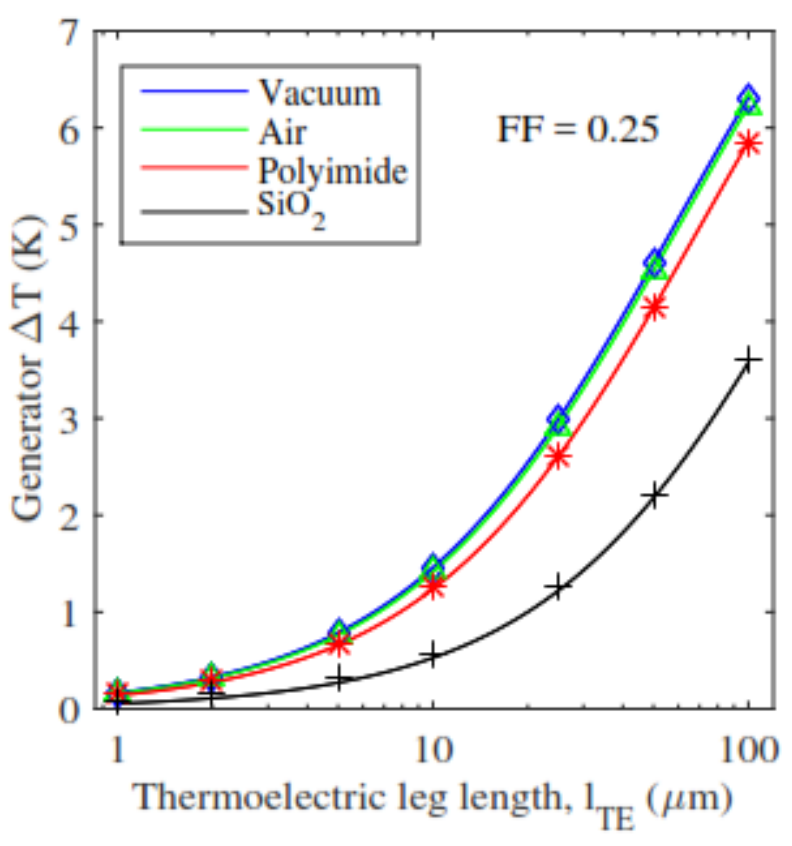

Fig. 3. Comparison of generator temperature difference calculated by the $1 D$ (lines) and 3D (markers) heat transfer models for (a) $F F=0.01$ and (b) $F F=0.25$ with different filler materials. 
The 1D thermal model neglecting the passivation layers and interconnects shows good agreement with the 3D finite element model. The $1 \mathrm{D}$ model begins to deviate from the $3 \mathrm{D}$ model for low fill fractions and thermoelectric leg lengths on the order of the interconnect height.

The electric circuit equations are coupled to the thermal model through the junction temperatures $T_{\mathrm{TEG}, \mathrm{h}}$ and $T_{\mathrm{TEG}, \mathrm{c}}$, which determine the generated Seebeck voltage $V_{\mathrm{gen}}$ :

$$
V_{\mathrm{gen}}=n_{\mathrm{TC}} S_{\mathrm{net}}\left(T_{\mathrm{TEG}, \mathrm{h}}-T_{\mathrm{TEG}, \mathrm{c}}\right)
$$

The voltage drops across the generator resistance $R_{\mathrm{e}, \mathrm{TEG}}$, which includes contact and interconnect (IC) resistances, and the connected load resistance $R_{\mathrm{e}, \text { load }}$ are then found using voltage divider expressions:

$$
\begin{aligned}
V_{\mathrm{TEG}} & =\frac{R_{\mathrm{e}, \mathrm{TEG}}}{R_{\mathrm{e}, \mathrm{TEG}}+R_{\mathrm{e}, \text { load }}} V_{\mathrm{gen}} \\
V_{\text {load }} & =\frac{R_{\mathrm{e}, \text { load }}}{R_{\mathrm{e}, \mathrm{TEG}}+R_{\mathrm{e}, \mathrm{load}}} V_{\mathrm{gen}}
\end{aligned}
$$

The electric current $I$ is calculated as the generated Seebeck voltage divided by the electrical resistance of the total pathway through generator and load:

$$
I=\frac{V_{\text {gen }}}{R_{\mathrm{e}, \mathrm{TEG}}+R_{\mathrm{e}, \text { load }}}
$$

The electric power delivered to the load is then calculated using the voltage across the load resistance given by Eq. (6) to write:

$$
P_{\text {load }}=\frac{V_{\text {load }}^{2}}{R_{\text {elload }}}=\frac{R_{\text {elload }} n_{\mathrm{TC}}^{2} S_{\text {net }}^{2}\left(T_{\mathrm{TEG}, \mathrm{h}}-T_{\mathrm{TEG}, \mathrm{c}}\right)^{2}}{\left(R_{\mathrm{e}, \mathrm{TEG}}+R_{\mathrm{e}, \text { load }}\right)^{2}}
$$

The electric power and the voltage and current produced by the generator are of primary interest, and the coupled system of equations (2-8) requires an iterative solution to obtain the temperatures on the hot and cold active regions of the generator, $T_{\mathrm{TEG}, \mathrm{h}}$ and $T_{\mathrm{TEG}, \mathrm{c}}$, respectively. 
The fraction of temperature drop across the thermoelectric versus the total available temperature difference from the reservoirs is less than unity for nonzero external thermal resistances and can be quantified as a temperature efficiency that is useful for device analysis:

$$
\eta_{\mathrm{T}}=\frac{T_{\mathrm{TEG}, \mathrm{h}}-T_{\mathrm{TEG}, \mathrm{c}}}{T_{\mathrm{h}}-T_{\mathrm{c}}}
$$

\section{Closed-form solution for temperature}

The coupled system described by Eqs. (2-8) requires an iterative numerical approach to converge to a solution. However, simplifications can be used to obtain a closed-form solution. One firstorder approach [17,21] neglects both the Peltier and Joule heating contributions and calculates the relevant temperature difference from a ratio of thermal resistances analogous to a voltage divider:

$$
\left(T_{\mathrm{TEG}, \mathrm{h}}-T_{\mathrm{TEG}, \mathrm{c}}\right)_{1 \mathrm{st}-\text { order }}=\frac{\left(\frac{1}{R_{\mathrm{t}, \mathrm{TE}}}+\frac{1}{R_{\mathrm{t}, \mathrm{filler}}}\right)^{-1}}{R_{\mathrm{t}, \mathrm{h}}+\left(\frac{1}{R_{\mathrm{t}, \mathrm{TE}}}+\frac{1}{R_{\mathrm{t}, \text { filler }}}\right)^{-1}+R_{\mathrm{t}, \mathrm{c}}}\left(T_{\mathrm{h}}-T_{\mathrm{c}}\right)
$$

The resulting relation requires no numerical iteration, but leads to a non-negligible error, on the order of $10 \%$, primarily due to absence of the Peltier heats. We obtain a more accurate closedform expression by retaining a form of the Peltier heating term. In the domain of small temperature differences the conversion efficiency is very small due to the theoretical limits of heat engine thermal efficiency. This allows us to neglect the effect of Joule heating in Eqs. (2) and (3), which is negligible compared to the total heat flow in the device ( $\sim 3 \%$ for a Carnot 
engine operating between 20 and 30 C). If Eqs. (2) and (3) are then added and combined with Eqs. (4) and (7), the following equation is produced:

$$
\begin{aligned}
& \frac{T_{\mathrm{h}}-T_{\mathrm{TEG}, \mathrm{h}}}{R_{\mathrm{t}, \mathrm{h}}}+\frac{T_{\mathrm{TEG}, \mathrm{c}}-T_{\mathrm{c}}}{R_{\mathrm{t}, \mathrm{c}}}=2 \frac{T_{\mathrm{TEG}, \mathrm{h}}-T_{\mathrm{TEG}, \mathrm{c}}}{R_{\mathrm{t}, \mathrm{TEG}}}+2 \frac{T_{\mathrm{TEG}, \mathrm{h}}-T_{\mathrm{TEG}, \mathrm{c}}}{R_{\mathrm{t}, \text { filler }}}+\ldots \\
& \frac{n_{\mathrm{TC}}^{2} S_{\mathrm{net}}^{2}\left(T_{\mathrm{TEG}, \mathrm{h}}-T_{\mathrm{TEG}, \mathrm{c}}\right)}{R_{\mathrm{e}, \text { load }}+R_{\mathrm{e}, \mathrm{TEG}}}\left(T_{\mathrm{TEG}, \mathrm{h}}+T_{\mathrm{TEG}, \mathrm{c}}\right)
\end{aligned}
$$

For small temperature difference near room temperature, such as seen by microscale generators in waste heat recovery applications, it can be assumed with reasonable accuracy that $\left(T_{\mathrm{TEG}, \mathrm{h}}+\right.$ $\left.T_{\mathrm{TEG}, \mathrm{c}}\right) \approx\left(T_{\mathrm{h}}+T_{\mathrm{c}}\right)$, which approximates the Peltier heat contribution in terms of the known reservoir temperatures. These temperatures are assumed constant due to the small amount of heat extracted by a $\mu$ TEG compared to the heat capacity of a hot wall or pipe, for example. In a final step, it is assumed that the external thermal resistances $R_{\mathrm{t}, \mathrm{h}}$ and $R_{\mathrm{t}, \mathrm{c}}$ are expressible as an average $R_{\mathrm{t}, \text { ext,avg. }}$ With these steps, a closed-form solution for the temperature difference across the active region of the generator is derived:

$$
\left(T_{\mathrm{TEG}, \mathrm{h}}-T_{\mathrm{TEG}, \mathrm{c}}\right)_{\text {closed }}=\frac{T_{\mathrm{h}}-T_{\mathrm{c}}}{1+R_{\mathrm{t}, \mathrm{ext}, \mathrm{arg}}\left[\frac{2}{R_{\mathrm{t}, \mathrm{TEG}}}+\frac{2}{R_{\mathrm{t}, \mathrm{filler}}}+\frac{n^{2} S_{\mathrm{net}}^{2}}{R_{\mathrm{e}, \text { load }}+R_{\mathrm{e}, \mathrm{TEG}}}\left(T_{\mathrm{h}}+T_{\mathrm{c}}\right)\right]}
$$

The temperature difference calculated in Eq. (12) can then be used directly in Eq. (8) to determine electric power delivered to a resistive load. Two limiting cases for Eq. (12) can be considered to verify physical intuition. First, as $R_{\mathrm{t} \text { ext,avg }} \rightarrow 0 \mathrm{~K} \mathrm{~W}^{-1}$, the case of no thermal resistance between the generator junctions and the thermal reservoirs, the temperature efficiency $\eta_{\mathrm{T}} \rightarrow 1$, and the junction temperature difference $\left(T_{\mathrm{TEG}, \mathrm{h}}-T_{\mathrm{TEG}, \mathrm{c}}\right)$ simplifies to the reservoir temperature difference $\left(T_{\mathrm{h}}-T_{\mathrm{c}}\right)$. Second, as $R_{\mathrm{t}, \text { ext,avg }} \rightarrow \infty \mathrm{K} \mathrm{W}^{-1}$, the case of external thermal resistances much greater than the generator and filler thermal resistance, the temperature 
efficiency $\eta_{\mathrm{T}} \rightarrow 0$ and the junction temperature difference $\left(T_{\mathrm{TEG}, \mathrm{h}}-T_{\mathrm{TEG}, \mathrm{c}}\right)$ approaches $0 \mathrm{~K}$ because effectively all of the available temperature difference is dropped across the external resistances.

The closed-form model, Eq. (12), can be compared quantitatively with the iterative, Eqs. (2-3), and $1^{\text {st }}$ order, Eq. (10), models by considering a device with nominal thermophysical properties of thermoelectric material comparable to bismuth telluride compounds, given in Table 1. Thermoelectric legs for the model are square in cross-section (side length $15 \mu \mathrm{m}$ ) with a fill fraction of $F F=0.01$. The fill fraction of vertically-aligned thermoelectric devices is defined to be the fraction of $A_{\text {total }}$ occupied by active thermoelectric material. For electrical resistance calculations, interconnects are modeled as gold with width equal to the width of the adjacent thermoelectric leg, and thickness of $1 \mu \mathrm{m}$. Filler material is air with thermal conductivity of $0.024 \mathrm{~W} \mathrm{~m}^{-1} \mathrm{~K}^{-1}$ and negligible convective thermal transport inside the module.

Table 1. Input thermoelectric material parameters for one possible device configuration.

\begin{tabular}{lllll}
\hline & $\boldsymbol{S}\left(\boldsymbol{\mu} \mathbf{V ~ K ^ { - 1 } )}\right.$ & $\boldsymbol{\rho}(\boldsymbol{\Omega} \mathbf{~ m})$ & $\boldsymbol{k}\left(\mathbf{W} \mathbf{~ m}^{-1} \mathbf{K}^{-1}\right)$ & $Z T_{@ 300 \mathrm{~K}}$ \\
\hline p-type & 200 & $1.0 \mathrm{e}-5$ & 2.0 & 0.6 \\
\hline n-type & -200 & $1.0 \mathrm{e}-5$ & 2.0 & 0.6 \\
\hline
\end{tabular}

The values for $R_{\mathrm{t}, \mathrm{h}}$ and $R_{\mathrm{t}, \mathrm{c}}$ include thermal resistance through a $525 \mu \mathrm{m}$-thick monosilicon substrate as well as an external thermal resistance (i.e. heat exchanger) between the substrate and thermal reservoir. The nominal values for these external thermal resistances are $1 \times 10^{-5} \mathrm{~m}^{2} \mathrm{~K} \mathrm{~W}^{-1}$ to represent conduction from a solid hot-side heat reservoir (e.g. hot water pipe) and $1 \times 10^{-4} \mathrm{~m}^{2} \mathrm{~K}$ $\mathrm{W}^{-1}$ to represent fin-assisted natural convection to a cold-side reservoir (e.g. static air) at ambient conditions. These values are characteristic of a solid bond contact with spreading into a metal surface for the hot side and small commercially-available machined-aluminum fin arrays 
(approximately $10 \mathrm{~K} \mathrm{~W}^{-1}$ total thermal resistance), made area-specific using a nominal thermoelectric device footprint of $10 \mathrm{~mm}^{2}$. The results from the 1D iterative, closed-form, and first order approaches are compared in Fig. 4, using matched electrical loading conditions, where the load electrical resistance in Fig. $2 \mathrm{~b}$ is equal to the internal electrical resistance of the thermoelectric generator.

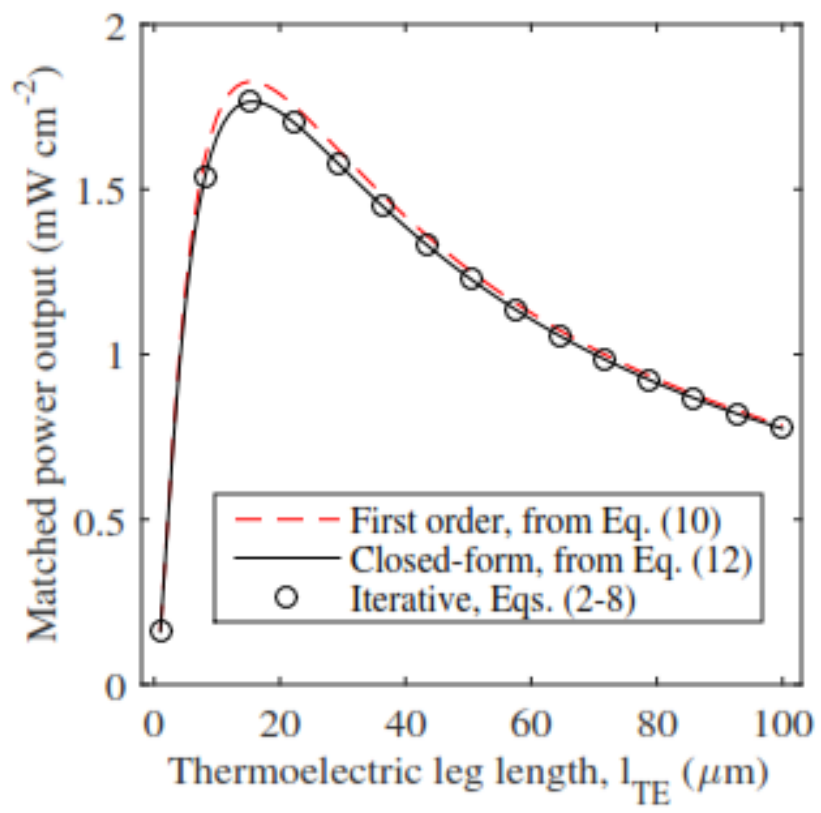

Fig. 4. Comparison of iterative 1D model (black squares calculated using Eqs. (2-8)), simplified first-order model (dashed red line calculated using Eqs. (10) and (8)), and the proposed closed-form model (solid black line calculated using Eqs. (12) and (8)).

The proposed closed-form solution shows excellent agreement with the iterative model and a noticeable improvement over the $1^{\text {st }}$ order model, particularly near maximum power conditions, where these devices are intended to operate. Nominal parameters used to produce simulation data are given in Table 2. We note that in the domain of microscale generators, electrical contact resistance in the series electrical pathway is significant and included, while thermal contact resistances in the parallel heat pathway are not and ignored.

Table 2. Nominal simulation parameters. 


\begin{tabular}{ll}
\hline Parameter & Nominal value \\
\hline Net Seebeck coefficient, $S_{\text {net }}$ & $400 \mu \mathrm{K} \mathrm{K}^{-1}$ \\
\hline Thermoelectric material thermal conductivity, $k_{\mathrm{TE}}$ & $2 \mathrm{~W} \mathrm{~m}^{-1} \mathrm{~K}^{-1}$ \\
\hline Thermoelectric material electrical resistivity, $\rho_{\mathrm{TE}}$ & $1.0 \times 10^{-5} \Omega \mathrm{m}$ \\
\hline Semiconductor/metal electrical contact resistance, $\rho_{\text {contact }}$ & $5.0 \times 10^{-11} \Omega \mathrm{m}^{2}[12,22]$ \\
\hline Cold reservoir temperature, $T_{\mathrm{c}}$ & $293.15 \mathrm{~K}$ \\
\hline Hot reservoir temperature, $T_{\mathrm{h}}$ & $303.15 \mathrm{~K}$ \\
\hline Total device area, $A_{\text {total }}$ & $1 \times 10^{-5} \mathrm{~m}^{2}$ \\
\hline Individual leg cross-sectional area, $A_{\text {leg }}$ & $2.25 \times 10^{-10} \mathrm{~m}^{2}$ \\
\hline Au interconnect thickness, $t_{\mathrm{IC}}$ & $1 \mu \mathrm{m}$ \\
\hline Area-specific thermal resistance to heat source, $R_{\mathrm{t}, \mathrm{h}}$ & $1 \times 10^{-5} \mathrm{~m}^{2} \mathrm{~K} \mathrm{~W}^{-1}$ \\
\hline Area-specific thermal resistance to heat sink, $R_{\mathrm{t}, \mathrm{c}}$ & $1 \times 10^{-4} \mathrm{~m}^{2} \mathrm{~K} \mathrm{~W}^{-1}$ \\
\hline Silicon substrate thickness & $525 \mu \mathrm{m}$ \\
\hline Si ${ }_{3} \mathrm{~N}_{4}$ passivation thickness & $100 \mathrm{~nm}$ \\
\hline
\end{tabular}

\section{Device design considerations}

Proper design of a thermoelectric generator for maximum power output relies on optimization of thermal resistance versus electrical resistance. To maximize electrical power from the generator, the thermal resistance of the active thermoelectric layer should be comparable to that of any inactive layers such as substrates and heat sinks. Increasing the ratio $R_{\mathrm{t}, \mathrm{TEG}} /\left(R_{\mathrm{t}, \mathrm{ext}, \mathrm{h}}+R_{\mathrm{t}, \mathrm{ext}, \mathrm{c}}\right)$ leads to a higher temperature efficiency, initially increasing the power through the ( $T_{\mathrm{TEG}, \mathrm{h}}-$ $\left.T_{\mathrm{TEG}, \mathrm{c}}\right)$ term in Eq. (8). However, the means of increasing this thermal resistance geometrically (i.e. by increasing leg length or decreasing leg cross-section) also increase the electrical resistance, which increases $R_{\mathrm{e}, \mathrm{TEG}}$. With all other parameters remaining constant, power output is maximized for a specific value of leg length or a specific value of fill fraction.

\subsection{Thermoelectric element length}

Changing the length of each thermoelectric element is a straightforward way to adjust the thermal and electrical resistance. Under fixed reservoir temperature conditions, an increase in leg length at first rapidly increases the temperature drop across the thermoelectric layer before the 
rate of change slows and asymptotically approaches the reservoir temperature difference $\left(\eta_{\mathrm{T}} \rightarrow\right.$ 1). In contrast, the electrical resistance continues to increase linearly with increasing leg length. These competing effects lead to a maximum, where we find an initial rise in power output due to rapidly increasing thermal resistance for short leg lengths followed by a decrease in power output due to the continuing increase in electrical resistance with diminishing benefits in thermal resistance. Key results from this work plotted vs. thermoelectric leg length, $l_{\mathrm{TE}}$, are given in Fig. 5. While the present analysis focuses on a small temperature differential, raising the source temperature $T_{\mathrm{h}}$ does not appreciably alter the optimal leg length.

(a)

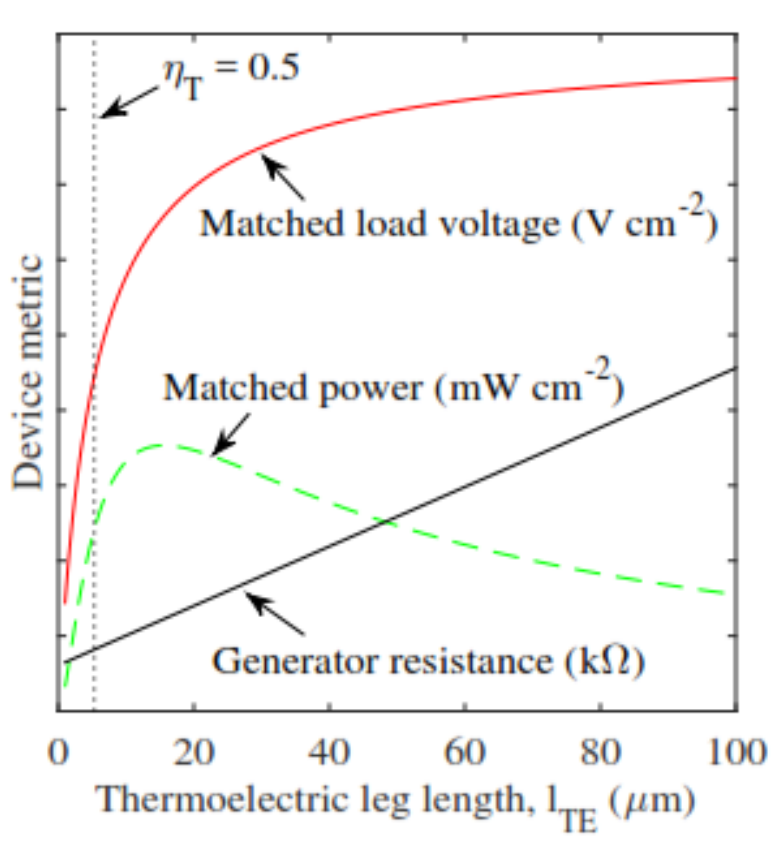

(c) (b)

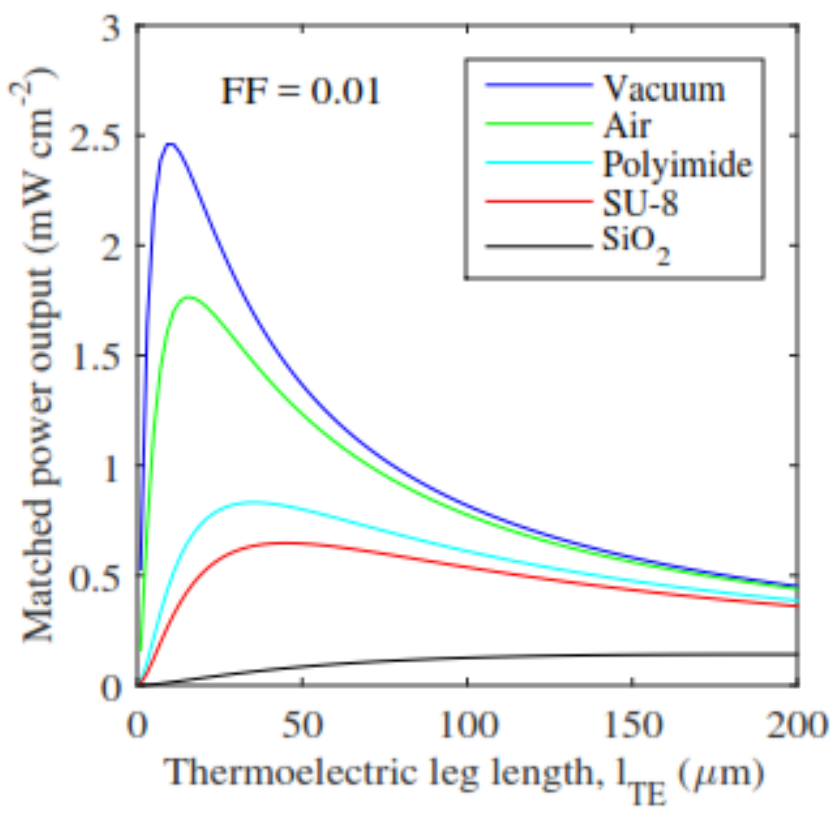

(d) 

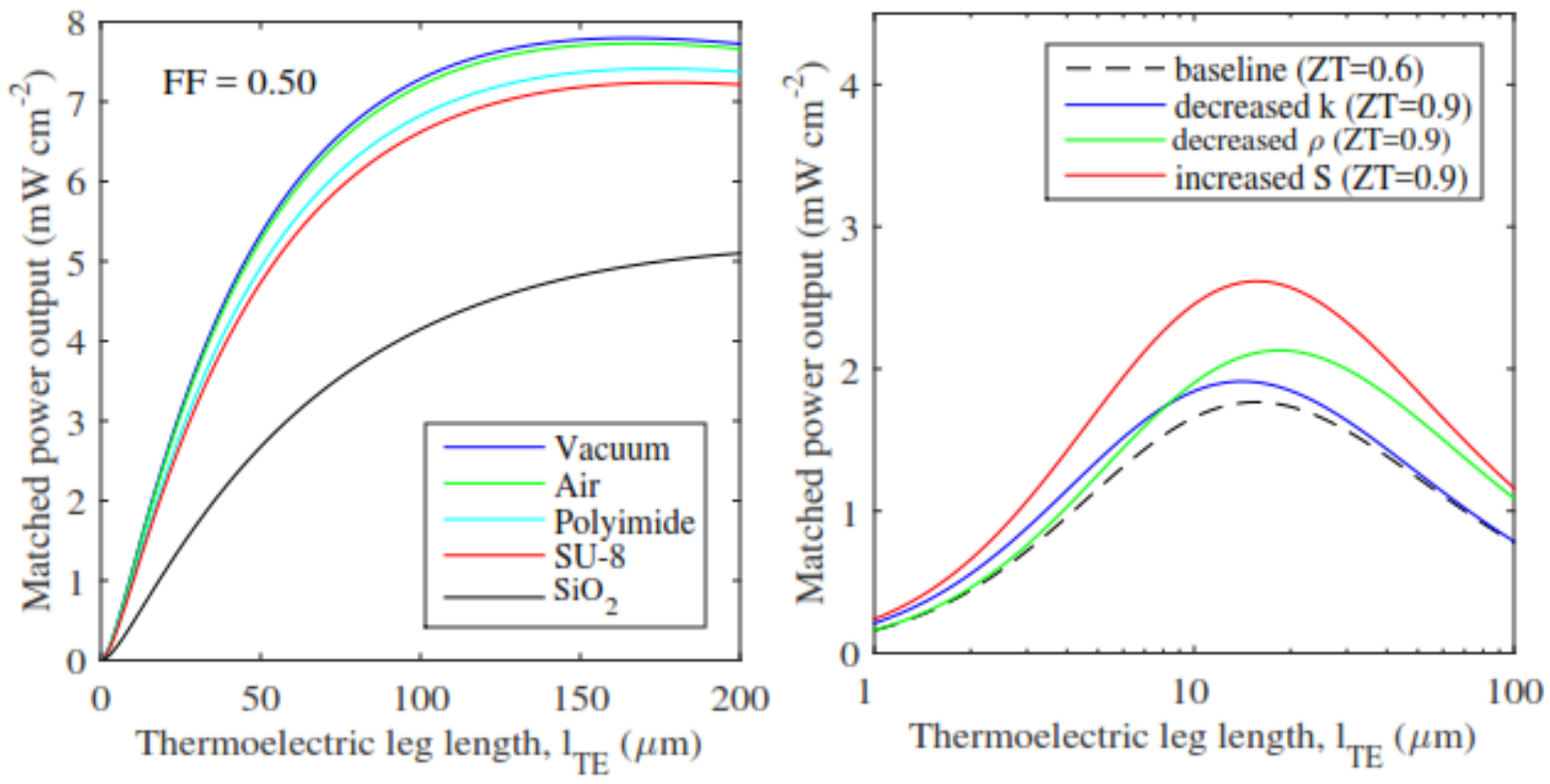

Fig. 5. Plot of (a) matched load voltage, load power, and device electrical resistance trends vs. thermoelectric leg length; matched power vs. leg length for (b) $F F=0.01$ and (c) $F F=$ 0.50 assuming different filler materials; (d) power vs. leg length curves calculated using closed-form solution for three different parameter combinations giving the same value of $Z T(0.9)$ at room temperature.

The competing temperature and electrical resistance effects are shown in Fig. 5a. In an ideal system, the maximum matched power is reached when half of the available reservoir temperature difference occurs across the thermoelectric layer $\left(\eta_{\mathrm{T}}=0.5\right)$. However, when parasitic losses such as interconnect electrical resistance are included as they are here, the target fractional temperature difference $\eta_{\mathrm{T}}$ changes, and the maximum power condition does in fact occur at a longer leg length than the $\eta_{\mathrm{T}}=0.5$ condition resulting in $\eta_{\mathrm{T} \text {,max power }}>0.5$. This observation is discussed in a later section of this work.

\subsection{Fill fraction}


The fabrication method imposes limits on maximum attainable leg length, particularly in the domain of microdevices constructed using bottom-up processes like sputtering or electrodeposition. This poses a constraint on the maximum thermal resistance that can be attained by simply extending the thermoelectric element. The fill fraction can be used to compensate for this limitation. While a very high fill fraction may seem desirable for efficient use of device real estate, it will be shown here that it is not desirable for power output except for very long elements. In fact, when element lengths are limited to $\sim 1-10 \mu \mathrm{m}$ as is common with current thin film technology, optimal fill fractions are very low $(<10 \%)$.

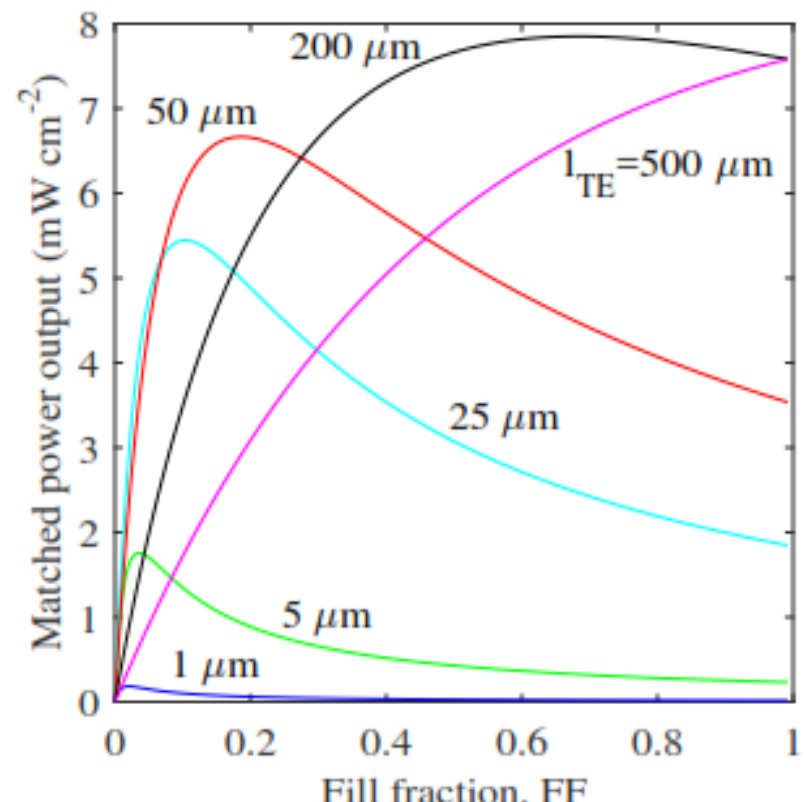

\section{Fig. 6. Matched power per-unit-area vs. fill fraction for different thermoelectric element lengths, using the results of the closed-form solution from Eq. (12).}

The impact of thermoelectric leg length and device fill fraction (varied by changing $n_{\mathrm{TC}}$ with constant element cross section) on power output is illustrated in Fig. 6. The data show that a maximum power output exists at some fill fraction for a fixed leg length, and that for very short leg lengths, this optimum fill fraction approaches zero. At very long leg lengths, the maximum 
would appear to occur for $F F>1$, which is non-physical. The thermal resistance due to the thermoelectric under these conditions is too large to reach the maximum power condition with any fill fraction. This motivates the thin-film fabrication approach, as optimal leg lengths for achievable fill fractions may be much shorter than what is possible with bulk machining processes. As seen in Fig. 6, the maximum attainable power output occurs when higher $F F$ is used in combination with longer leg length, as long as their combined effect on thermal resistance remains optimal. It is important to note here that the maximum power output for each leg length would be identical, though still occurring for different fill fractions, if electrical losses due to the interconnects and thermal shunting due to the filler material were neglected. It will be shown that increasing fill fraction by increasing the number of thermocouples reduces the parasitic effect of non-ideal interconnects.

Fill fraction also has an effect on the sensitivity of the optimal leg length to the reservoir temperature gradient. Increasing the fill fraction leads to a longer optimal leg length, as shown in Fig. 6, and at this leg length, a larger electric power generation. The resulting larger Peltier heats diminish the steady state junction temperature difference, and a longer leg length must be reached to compensate. This effect is shown for two fill fractions, 0.01 and 0.50 , in Fig. 7. The plots also show an increasing error with increased temperature gradient at higher fill fractions due to the approximation made for the Peltier heat contribution in the closed-form equation, but results in only a $2.3 \%$ difference in calculated optimal leg length for air filler, $50 \%$ fill fraction, and $100 \mathrm{~K}$ available reservoir temperature difference. 

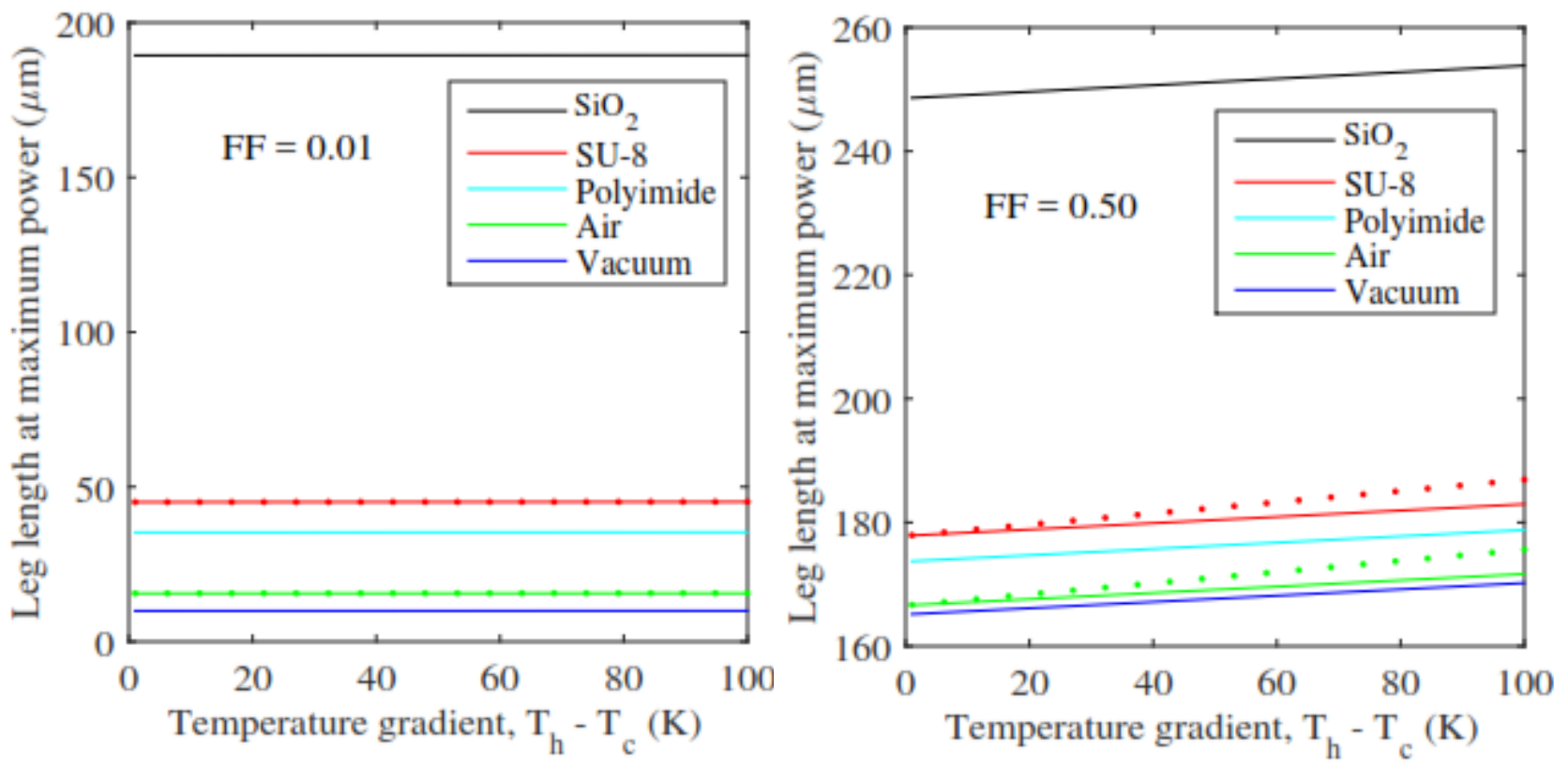

Fig. 7. Dependence of optimal leg length on reservoir temperature gradient for fill fractions of (a) 0.01 and (b) 0.50. Solid lines utilize the derived closed-form Eq. (12), while dotted lines calculated using the full iterative $1 D$ model are given for accuracy comparison.

If the Peltier heat contribution is removed from the equations, the optimal leg length is independent of temperature difference at any fill fraction. For significant temperature differences across the thermoelectric material, temperature-dependent properties will become important and further impact this relationship.

\subsection{Filler material}

When optimization leads to a very low fill fraction, the thermal properties of the filler material become critically important as the relatively large cross-sectional area may create a significant thermal shunt and reduce the temperature drop across the thermoelectric elements. The ideal filler material should have low thermal conductivity, considered here with a limiting case of vacuum $\left(k=0.0001 \mathrm{~W} \mathrm{~m}^{-1} \mathrm{~K}\right.$ from first-order kinetic theory calculations for air at $1 \mathrm{~Pa}, 300 \mathrm{~K}$, 
and a mean free path size-limiting dimension of $\sim 100 \mu \mathrm{m}$ ). In a real device this is not always a practical option as preservation of vacuum conditions means carefully sealing a $\mu \mathrm{m}$-scale structure and adding a competing thermal shunt through the seal. Air $\left(k=0.024 \mathrm{~W} \mathrm{~m}^{-1} \mathrm{~K}^{-1}\right)$ is a simple option, but oxidation can be a concern and it does not provide structural support during fabrication or operation. Polymers with low thermal conductivity such as polyimide $(k=0.14 \mathrm{~W}$ $\left.\mathrm{m}^{-1} \mathrm{~K}^{-1}\right)$ and SU-8 $\left(k=0.208 \mathrm{~W} \mathrm{~m}^{-1} \mathrm{~K}^{-1}\right)[12,21]$ have been used in an attempt to add structural integrity while minimizing thermal shunting, as opposed to the use of $\mathrm{SiO}_{2}\left(k=1.38 \mathrm{~W} \mathrm{~m}^{-1} \mathrm{~K}^{-1}\right)$ [16] which has a thermal conductivity comparable to bismuth telluride thermoelectric material.

Matched power per unit area vs. thermoelectric leg length is shown in Fig. $5 \mathrm{~b}$ and $\mathrm{c}$ for $F F=$ 0.01 and $F F=0.50$, respectively, and a variety of candidate filler materials. Each curve shows the same general trend: maximum matched power decreases and occurs at longer leg lengths with increasing filler thermal conductivity. With a more prominent thermal shunt (or large thermal gradients combined with moderate-to-high fill fractions), a longer leg length must be attained to reach the optimal temperature drop across the thermoelectric. This increased leg length increases electrical resistance and decreases power output. When the fill fraction is increased, two primary effects are seen. First, the impact of the specific filler material becomes less significant, and in fact, vacuum and air show nearly identical power curves at 0.50 fill fraction. This is simply due to the fact that there is less filler material, and its presence becomes negligible as $F F \rightarrow 1$. Second, higher maximum matched powers are reached, but at longer leg lengths. The reason that different maximum power outputs are reached is due to the reduction in parasitic losses, both thermal losses from a smaller thermal shunt and electrical losses from the interconnects. For low fill fractions, the thermal resistance is very high and the fraction of reservoir temperature difference dropped across the thermoelectric is large at short leg lengths. 
Hence, the electrical resistance due to the legs is relatively small, and parasitic losses through the interconnects contribute significantly. When fill fraction increases, the thermal resistance decreases, and a longer leg length must be reached to force the optimal temperature drop. The presence of a filler material with nonzero thermal conductivity will reduce the parallel thermal resistance. The following relationship appropriately matches the filler and thermoelectric thermal conductivities based on fill fraction $F F$ and maximum desired thermal resistance reduction factor $F_{\mathrm{R}, \max }:$

$$
\frac{k_{\text {filler }}}{k_{\mathrm{TE}}} \leq F_{\mathrm{R}, \max } \frac{F F}{1-F F}
$$

For example, with a fill fraction of 0.1 and a desired maximum thermal resistance reduction (from perfect vacuum filler conditions) of 0.1 (or 10\%), materials should be selected such that $k_{\text {filler }} \leq 0.011 k_{\mathrm{TE}}$.

\subsection{Number of thermocouples}

Another design consideration is the selection of the number of thermocouples. The fill fraction is non-unique in that the total area occupied by thermoelectric material depends on both the number

of legs and the cross-sectional area of each leg. Therefore multiple combinations of thermocouple number density and individual leg cross-section will result in the same fill fraction, as illustrated in Fig. 8. 


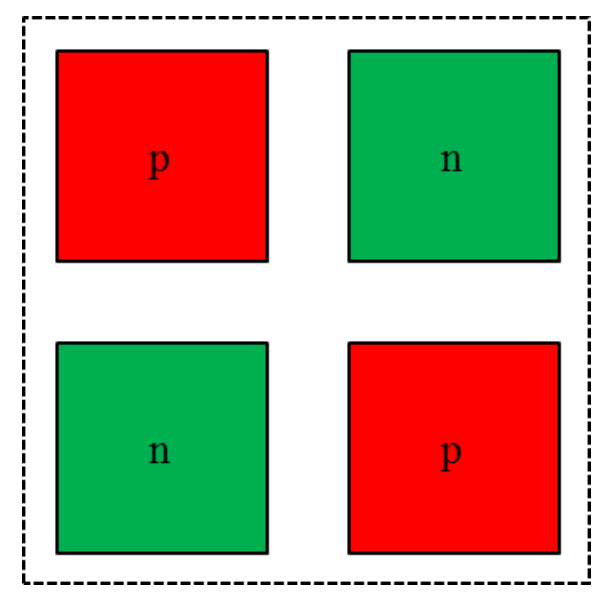

(b)

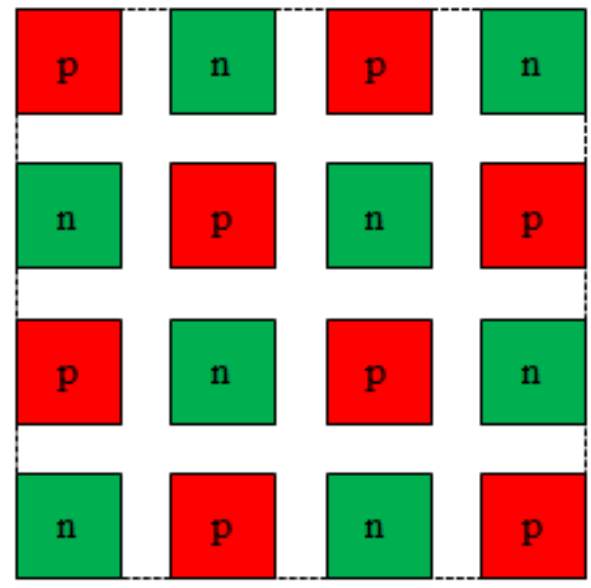

Fig. 8. Configurations with (a) $n_{\mathrm{TC}}=2$ and (b) $n_{\mathrm{TC}}=8$ each resulting in the same fill fraction, with different numbers of thermocouples and leg cross-section.

Under ideal conditions (i.e. no parasitic thermal or electrical losses), matched power output will be identical for any combination of thermocouple count and leg cross-section which results in the same fill fraction for the same total area. Beginning with the definition of fill fraction as the ratio of thermoelectric material cross sectional area to total footprint:

$$
F F=\frac{2 n_{\mathrm{TC}} A_{\text {leg }}}{A_{\text {total }}} \rightarrow A_{\text {leg }}=\frac{A_{\text {total }} F F}{2 n_{\mathrm{TC}}}
$$


Under matched loading conditions, the voltages dropped across the generator and the load resistance are equivalent:

$$
V_{\text {matched }}=\frac{n_{\mathrm{TC}} S_{\mathrm{net}}\left(T_{\mathrm{TEG}, \mathrm{h}}-T_{\mathrm{TEG}, \mathrm{c}}\right)}{2}
$$

The electrical resistance of the series of thermoelectric elements not accounting for parasitic interconnect or contact resistance, and therefore the load for matched loading conditions, can be related to the fill fraction $F F$ :

$$
R_{\mathrm{e}}=2 n_{\mathrm{TC}} \rho_{\mathrm{TE}} \frac{l_{\mathrm{TE}}}{A_{\mathrm{leg}}}=4 n_{\mathrm{TC}}^{2} \frac{\rho_{\mathrm{TE}} l_{\mathrm{TE}}}{A_{\mathrm{total}} F F}
$$

We then calculate the power delivered to the load using Eqs. (14b) and (14c):

$$
P_{\text {matched }}=\frac{V_{\text {matched }}^{2}}{R_{\mathrm{e}}}=\frac{A_{\mathrm{total}} S_{\mathrm{net}}^{2} F F\left(T_{\mathrm{TEG}, \mathrm{h}}-T_{\mathrm{TEG}, \mathrm{c}}\right)^{2}}{16 \rho_{\mathrm{TE}} l_{\mathrm{TE}}}
$$

With fill fraction constant, the thermal resistance is unchanged. The Peltier and Joule heating terms are the only remaining parameters in the heat balance which could affect the matched power calculation. The electrical current in terms of the number of thermocouples $n_{\mathrm{TC}}$ is:

$$
I=\frac{V_{\text {matched }}}{R_{\mathrm{e}}}=\frac{A_{\mathrm{total}} S_{\mathrm{net}} F F\left(T_{\mathrm{TEG}, \mathrm{h}}-T_{\mathrm{TEG}, \mathrm{c}}\right)}{8 n_{\mathrm{TC}} \rho_{\mathrm{TE}} l_{\mathrm{TE}}}
$$

The Peltier heating contributions at the hot and cold junctions are dependent on fill fraction $F F$, but independent of the number of thermocouples $n_{\mathrm{TC}}$ :

$$
\dot{Q}_{\mathrm{Peltier}, \mathrm{h}}=-n_{\mathrm{TC}} S_{\mathrm{net}} I T_{\mathrm{TEG}, \mathrm{h}}=\frac{-T_{\mathrm{TEG}, \mathrm{h}} A_{\mathrm{total}} S_{\mathrm{net}}^{2} F F\left(T_{\mathrm{TEG}, \mathrm{h}}-T_{\mathrm{TEG}, \mathrm{c}}\right)}{8 \rho_{\mathrm{TE}} l_{\mathrm{TE}}}
$$




$$
\dot{Q}_{\text {Peltier, },}=\frac{T_{\text {TEG, },} A_{\text {total }} S_{\text {net }}^{2} F F\left(T_{\text {TEG, h }}-T_{\text {TEG, } \mathrm{c}}\right)}{8 \rho_{\mathrm{TE}} l_{\mathrm{TE}}}
$$

Under matched electrical loading, the power delivered to the load is equivalent to the power dissipated in the generator by Joule heating.

For matched load conditions and constant total area, the heat balance equations will not change for any combination of thermocouple number and leg cross-section that gives the same fill fraction. In the absence of parasitic losses, this property can be used to design the generator for a desired voltage output or electrical resistance. Increasing the number of thermocouples should be the preferred approach to reach a target load voltage when expected source/sink temperatures are known, as voltage boost circuits may result in significant electrical conversion penalties.

$$
n_{\mathrm{TC}}=\frac{V_{\text {gen,desired }}}{S_{\text {net }}\left(T_{\mathrm{TEG}, \mathrm{h}}-T_{\mathrm{TEG}, \mathrm{c}}\right)}
$$

If parasitic losses are considered, particularly due to electrical resistance over the interconnect length between each thermoelectric element and contact resistance at the semiconductor-metal junctions, the power output may vary with different combinations of element area and number of thermocouples. The electrical resistance along total length of all interconnects within the device is derived geometrically as:

$$
R_{\text {e,total, }, \mathrm{CC}}=2 n_{\mathrm{TC}} \rho_{\mathrm{IC}} \frac{\sqrt{\frac{A_{\mathrm{TE}}}{F F}}+\sqrt{A_{\mathrm{TE}}}}{t_{\mathrm{IC}} \sqrt{A_{\mathrm{TE}}}}
$$

Utilizing the generator electrical resistance from Eq. (14c) and calculating the ratio of parasitic to generator electrical resistance: 


$$
\frac{R_{\mathrm{e}, \text { total, IC }}+R_{\mathrm{e}, \text { total,contact }}}{R_{\mathrm{e}, \mathrm{tota}, \mathrm{TE}}}=\frac{\rho_{\mathrm{IC}} \frac{\sqrt{\frac{1}{F F}}+1}{t_{\mathrm{IC}}}+2 \frac{\rho_{\text {contact }}}{A_{\text {leg }}}}{\rho_{\mathrm{TE}} \frac{l_{\mathrm{TE}}}{A_{\mathrm{leg}}}}=\frac{A_{\mathrm{total}} F F \rho_{\mathrm{IC}}\left(\sqrt{\frac{1}{F F}}+1\right)}{2 n_{\mathrm{TC}} \rho_{\mathrm{TE}} t_{\mathrm{IC}} l_{\mathrm{TE}}}+2 \frac{\rho_{\text {contact }}}{\rho_{\mathrm{TE}} l_{\mathrm{TE}}}
$$

Equation (17b) shows that when keeping fill fraction constant the parasitic electrical resistance of the interconnects and semiconductor/metal junctions relative to the resistance of active thermoelectric material is related to the inverse of the number of thermocouples. Once the fill fraction has been chosen for optimal thermal resistance, it is beneficial to utilize many thermocouples with smaller cross-sectional area. This is typically convenient as increasing the number of thermocouples will increase the output Seebeck voltage from the device. The legs should also be as long as possible to minimize the relative impact of contact resistance.

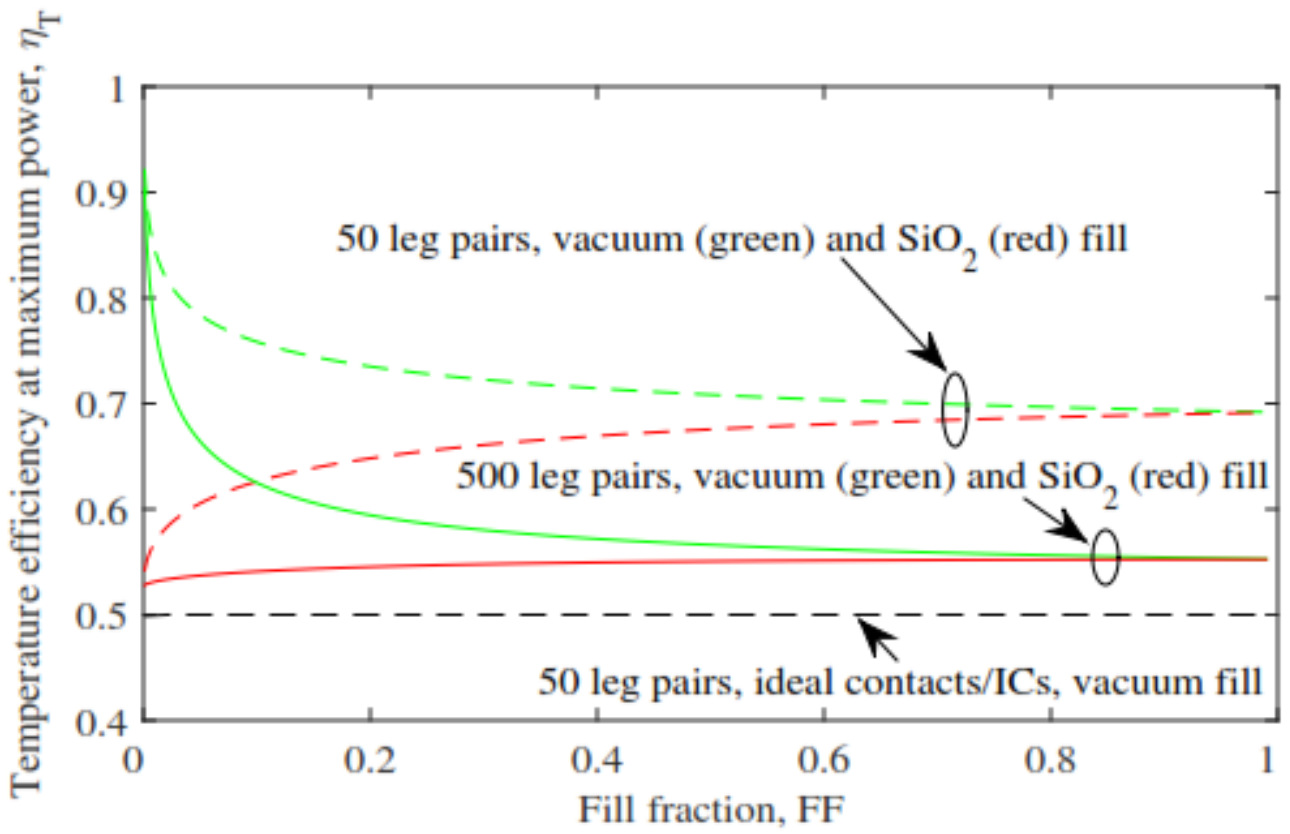

Fig. 9. Temperature efficiency at maximum matched power output vs. fill fraction, where fill fraction is adjusted by changing the area of thermoelectric elements with constant $\eta_{\mathrm{TC}}$ and $t_{\mathrm{IC}}$ is the interconnect thickness (or height). At each fill fraction value, the thermoelectric leg length $l_{\mathrm{TE}}$ is adjusted to reach maximum matched power. 
The required temperature efficiency for maximum matched power versus fill fraction is shown in Fig. 9 for different numbers of thermoelectric leg pairs and filler materials in a $10 \mathrm{~mm}^{2}$ footprint. In the idealized case of interconnects with infinite electrical conductance, no electrical contact resistance, and negligible parasitic thermal losses, the optimal temperature drop is always half of the available temperature difference from the thermal reservoirs, as discussed in the literature [18]. When parasitic losses are included, a larger thermal resistance (i.e. longer leg length) must be reached to compensate. Furthermore, the required temperature efficiency for maximum matched power is significantly higher than the ideal temperature efficiency when the thermal conductivity of the filler material is very low. This demonstrates a weakness of the vast majority of previous system analyses, which not only neglect the impact of interconnect and contact resistance but assume no contribution from the filler material, (i.e. assuming no parasitic interconnect resistance and the case for which this resistance is most significant). The concavity of the curve depends on the ratio of thermal resistance of the filler region to that of the active thermoelectric elements. If the filler material has very low conductivity, then the thermal shunt through filler material is very small even at low fill fractions, and it is beneficial to have a higher fraction of the available temperature difference across the active thermoelectric materials. Conversely, for high filler material conductivities there is a significant thermal shunt through the filler region, particularly at low fill fractions. In this case it is less desirable to have a large fraction of the available temperature difference dropped across the filler/thermoelectric.

The varying optimal temperature efficiency is a factor of the parasitic loss introduced by the interconnect electrical resistance since the geometry (e.g. leg length, fill fraction, etc.) must be altered to give a larger temperature difference, and the resulting increased Seebeck voltage compensates for the additional voltage drops through the series circuit. Inclusion of the Peltier 
heat contribution also affects the optimal temperature efficiency, although less significantly. If it is neglected as in the first order approximation [17,21], the optimal temperature efficiency is even higher, as that temperature-dependent loss is no longer contributing to the thermal equation.

\subsection{Incompleteness of $Z T$}

Many analyses suggest that the magnitude of the parameter $Z T$, regardless of its composition, is the ultimate rule to device performance. However, due to the optimization balance of thermal and electrical resistance and the concurrent presence of thermal and electrical conductivity in $Z$, the optimal device design will be different depending on the ratio of these parameters even for identical values of $Z$.

Table 3. Maximum matched power and corresponding TE element leg length for a 50\% increase in $Z T$ by modifying each parameter of $Z$ individually.

\begin{tabular}{cccccc}
\hline $\boldsymbol{k}_{\mathrm{TE}}(\mathbf{W} / \mathbf{m}-\mathbf{K})$ & $\boldsymbol{\rho}_{\mathrm{TE}}(\mathbf{\Omega}-\mathbf{m})$ & $\boldsymbol{S}_{\text {net }}(\boldsymbol{\mu} \mathbf{V} / \mathbf{K})$ & $\boldsymbol{Z T}$ @ 300K & $\begin{array}{c}\boldsymbol{P}_{\text {matched,max }} \\
\left(\mathbf{m W / \mathbf { m } ^ { 2 }}\right)\end{array}$ & $\boldsymbol{l}_{\text {TE,max }}(\boldsymbol{\mu m})$ \\
\hline 1.325 & $1 \mathrm{e}-5$ & 400 & 0.9 & 1.91 & 14.1 \\
\hline 2 & $6.63 \mathrm{e}-6$ & 400 & 0.9 & 2.13 & 18.5 \\
\hline 2 & $1 \mathrm{e}-5$ & 491 & 0.9 & 2.62 & 15.7 \\
\hline
\end{tabular}

The data in Fig. 5d and Table 3 reveal key points to consider for thermoelectric device design and materials selection. First, the tuning of individual material parameters does not give equivalent device performance, even if they result in the same $Z T$. This is due to the competing interaction between thermal and electrical resistance and how they differ according to device design. Second, depending on the attainable film thickness, it may be beneficial to optimize one parameter over another, if possible. While increasing the Seebeck coefficient consistently increases the performance in this example more than changes in $k$ or $\rho$ for a given design, if the 
leg lengths are very short (e.g. less than about $5 \mu \mathrm{m}$ in Fig. $5 \mathrm{~d})$ it is better to reduce thermal conductivity than to increase electrical conductivity. If longer leg lengths are attainable, the opposite is true. Finally, the optimization of the different parameters can lead to a shift in the optimal thermoelectric leg length. While increasing $S$ does not alter the condition of maximum matched power, a decrease in $k$ results in a decreased optimal leg length. This is because the thermal resistance of the device is increased and the ideal temperature drop across the thermoelectric elements can be achieved without adding increased electrical resistance. Conversely, when the device is optimized by increasing electrical conductivity the leg length at maximum matched power is increased. This is because a longer leg length can be reached to realize an optimal temperature drop before added electrical resistance from increased leg length becomes dominant.

These trends become even more impactful when examined in multi-parameter space. A contour plot of maximum matched power for varying thermal conductivity and net Seebeck coefficient is given in Fig. 10. Electrical resistivity and fill fraction each remain fixed to nominal values, and the leg length is adjusted at each data point to maximize the matched power output. Iso-ZT lines are shown in red, while two isopower lines are provided in black indicating 1.0 and $2.0 \mathrm{~mW} \mathrm{~cm}^{-}$ ${ }^{2}$. As one example from this plot, the same maximum matched power of $2.0 \mathrm{~mW} \mathrm{~cm}{ }^{-2}$ can be reached in a device with $Z T$ values of 0.6 and 1.4 within this parametric space. The variable system parameters for each point (indicated with a black box marker) are $S_{\mathrm{net}}=435.4 \mu \mathrm{V} \mathrm{K}{ }^{-1}, k_{\mathrm{TE}}$ $=2.36 \mathrm{~W} \mathrm{~m}^{-1} \mathrm{~K}^{-1}, l_{\mathrm{TE}, \max }=16.4 \mu \mathrm{m}(\mathrm{ZT}=0.5987)$, and $S_{\mathrm{net}}=396.0 \mu \mathrm{V} \mathrm{K}^{-1}, k_{\mathrm{TE}}=0.822 \mathrm{~W} \mathrm{~m}^{-1} \mathrm{~K}^{-}$ ${ }^{1}, l_{\mathrm{TE}, \max }=13.0 \mu \mathrm{m}(Z T=1.422)$. 


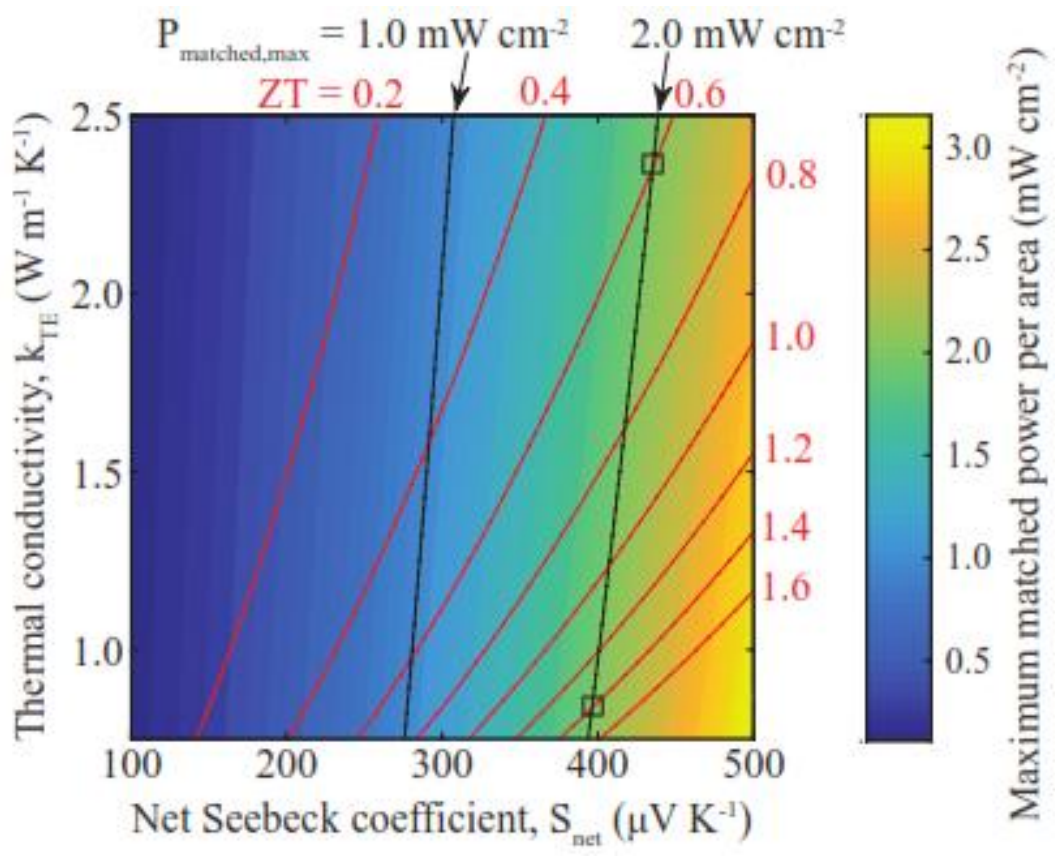

Fig. 10. Contour plot of maximum matched power per area for ranges of thermal conductivity and net Seebeck coefficient. $Z T$ values are included as red isolines for reference, and two black isopower lines are included at 1.0 and $2.0 \mathrm{~mW} \mathrm{~cm}$.

These findings are critical for device design decisions since a review of thermoelectric literature reveals an abundance of focus on tuning only $Z T$ values for material and device performance.

\section{Summary and Concluding Remarks}

This work derives a closed-form solution that closely matches the coupled iterative solution for use in thermoelectric micro-generator modeling, targeting low-temperature waste heat recovery applications. The proposed formula yields a noticeable improvement in accuracy over simplified first-order models found in literature, while eliminating the need for numerical iteration in the coupled 1D heat transfer model. Additionally, the importance of often-neglected device design considerations has been illustrated, including fill fraction, filler material, number of thermocouples, and the inequality of the different parameters of the thermoelectric figure of 
merit $Z T$, focusing on matched power output under near-ambient fixed-temperature thermal reservoir conditions.

Key conclusions include the fact that a low fill fraction may be used to compensate for inadequate thermal resistance from fabrication-limited leg lengths. Furthermore, the transport properties of filler material may seriously impact the performance of devices fabricated with thin-film processing techniques. Increasing the number of thermocouples as much as possible for a given fill fraction is desirable to minimize parasitic losses due to the electrical interconnects. Finally, when attempting to increase $Z T$ for improved device performance, it is best to increase Seebeck coefficient, followed by decreasing thermal conductivity for short leg lengths and increasing electrical conductivity for long leg lengths. The reported model and observed trends can be used to guide design decisions for thermoelectric generators, particularly in the microscale thermal energy harvesting domain.

\section{Acknowledgements}

This work was supported by Analog Devices, Inc., as well as by the United States Department of Defense through the National Defense Science and Engineering Graduate Fellowship program.

\section{Nomenclature}

$\begin{array}{ll}A & \text { Area }\left(\mathrm{m}^{2}\right) \\ F_{\mathrm{R}, \max } & \text { Thermal resistance reduction factor } \\ F F & \text { Fill fraction }(-) \\ I & \text { Electric current }(\mathrm{A}) \\ k & \text { Thermal conductivity }\left(\mathrm{W} \mathrm{m}^{-1} \mathrm{~K}^{-1}\right) \\ l_{\mathrm{TE}} & \text { Thermoelectric leg length }(\mu \mathrm{m})\end{array}$




$\begin{array}{ll}n_{\mathrm{TC}} & \text { Number of thermocouple pairs } \\ \dot{Q} & \text { Heat rate }(\mathrm{W}) \\ R_{\mathrm{e}} & \text { Electrical resistance }(\Omega) \\ R_{\mathrm{t}} & \text { Thermal resistance }\left(\mathrm{K} \mathrm{W}^{-1}\right) \\ S & \text { Seebeck coefficient }\left(\mu \mathrm{V} \mathrm{K}^{-1}\right) \\ t & \text { Thickness }(\mu \mathrm{m}) \\ T & \text { Temperature }(\mathrm{K}) \\ V & \text { Electric voltage }(\mathrm{V}) \\ Z T & \text { Thermoelectric figure of merit }(-)\end{array}$

$\begin{array}{ll}\text { Greek } & \\ \eta_{\mathrm{T}} & \begin{array}{l}\text { Temperature efficiency } \\ \rho\end{array} \\ \text { Subscript } & \text { Electrical resistivity }(\Omega \mathrm{m}) \\ \mathrm{c} & \text { Cold side } \\ \text { filler } & \text { Related to material surrounding the thermoelectric material } \\ \text { gen } & \text { Generated by Seebeck effect } \\ \mathrm{h} & \text { Hot side } \\ \text { IC } & \text { Interconnect } \\ \text { Joule } & \text { Related to Joule heating effects } \\ \text { leg } & \text { Related to a single thermoelectric leg } \\ \text { load } & \text { Related to the connected electrical load } \\ \text { matched } & \text { Under matched electrical loading conditions } \\ \text { max } & \text { Corresponding to maximum power condition } \\ \text { net } & \text { Difference of } \mathrm{n} \text { - and p-type material properties } \\ \text { Peltier } & \text { Related to Peltier heating/cooling effects } \\ \text { TE } & \text { Related to the thermoelectric material } \\ \text { TEG } & \text { Related to the thermoelectric generator module } \\ \text { total } & \text { Related to the total device }\end{array}$

\section{References}

[1] H.P.J. De Bock, V. Novak, Evaluation of system configurations for thermoelectric power generation, in: 2008 11th IEEE Intersoc. Conf. Therm. Thermomechanical Phenom. Electron. Syst. I-THERM, 2008: pp. 1276-1282. doi:10.1109/ITHERM.2008.4544405.

[2] D. Rowe, G. Min, Evaluation of thermoelectric modules for power generation, J. Power Sources. 73 (1998) 193-198. 
[3] J. Meng, X. Zhang, X. Wang, Multi-objective and multi-parameter optimization of a thermoelectric generator module, Energy. 71 (2014) 367-376.

[4] J. Yang, T. Caillat, Thermoelectric materials for space and automotive power generation, MRS Bull. 31 (2006) 224-229.

[5] G. Min, D.M. Rowe, Optimisation of thermoelectric module geometry for "waste heat" electric power generation, J. Power Sources. 38 (1992) 253-259.

[6] J. Fleurial, A. Borshchevsky, T. Caillat, R. Ewell, New materials and devices for thermoelectric applications, in: Proc. 32nd Intersoc. Energy Convers. Eng. Conf., 1997: pp. 1080-1085.

[7] M. Stordeur, I. Stark, Low power thermoelectric generator - self-sufficient energy supply for micro systems, Proc. 16th Int. Conf. Thermoelectr. (1997) 575-577.

[8] J.P. Fleurial, G.J. Snyder, J.A. Herman, M. Smart, P. Shakkottai, P.H. Giauque, et al., Miniaturized Thermoelectric Power Sources, in: 34th Intersoc. Energy Convers. Eng. Conf. Proc., 1999.

[9] J.P. Fleurial, G.J. Snyder, J.A. Herman, P.H. Giauque, W.M. Phillips, M.A. Ryan, et al., Thick-Film Thermoelectric Microdevices, in: 18th Int. Conf. Thermoelectr., 1999: pp. 294-300.

[10] H. Böttner, J. Nurnus, A. Gavrikov, G. Kühner, M. Jägle, C. Künzel, et al., New thermoelectric components using microsystem technologies, J. Microelectromechanical Syst. 13 (2004) 414-420.

[11] M. Strasser, R. Aigner, C. Lauterbach, T.F. Sturm, M. Franosch, G. Wachutka, Micromachined CMOS thermoelectric generators as on-chip power supply, Sensors Actuators A Phys. 114 (2004) 362-370.

[12] W. Glatz, E. Schwyter, L. Durrer, C. Hierold, Bi2Te3-based flexible micro thermoelectric generator with optimized design, J. Microelectromechanical Syst. 18 (2009) 763-772.

[13] J. Carmo, Thermoelectric microconverter for energy harvesting systems, IEEE Trans. Ind. Electron. 57 (2010) 861-867.

[14] R. Vullers, R. Schaijk, Energy harvesting for autonomous wireless sensor networks, IEEE Solid-State Circuits Mag. (2010) 29-38.

[15] M. Takashiri, T. Shirakawa, K. Miyazaki, H. Tsukamoto, Fabrication and characterization of bismuth-telluride-based alloy thin film thermoelectric generators by flash evaporation method, Sensors Actuators A Phys. 138 (2007) 329-334. 
[16] Y. Li, K. Buddharaju, B.C. Tinh, N. Singh, S.J. Lee, Improved Vertical Silicon Nanowire Based Thermoelectric Power Generator With Polyimide Filling, IEEE Electron Device Lett. 33 (2012) 715-717.

[17] Y. Apertet, H. Ouerdane, O. Glavatskaya, C. Goupil, P. Lecoeur, Optimal working conditions for thermoelectric generators with realistic thermal coupling, EPL (Europhysics Lett. 97 (2012) 28001.

[18] K. Yazawa, A. Shakouri, Optimization of power and efficiency of thermoelectric devices with asymmetric thermal contacts, J. Appl. Phys. 111 (2012) 024509.

[19] C.-C. Wang, C.-I. Hung, W.-H. Chen, Design of heat sink for improving the performance of thermoelectric generator using two-stage optimization, Energy. 39 (2012) 236-245.

[20] M. Strasser, R. Aigner, M. Franosch, G. Wachutka, Miniaturized thermoelectric generators based on poly-Si and poly-SiGe surface micromachining, Sensors Actuators A Phys. 97-98 (2002) 535-542.

[21] W. Glatz, S. Muntwyler, C. Hierold, Optimization and fabrication of thick flexible polymer based micro thermoelectric generator, Sensors Actuators A Phys. 132 (2006) $337-345$.

[22] L.W. da Silva, M. Kaviany, Micro-thermoelectric cooler: interfacial effects on thermal and electrical transport, Int. J. Heat Mass Transf. 47 (2004) 2417-2435. doi:10.1016/j.ijheatmasstransfer.2003.11.024. 